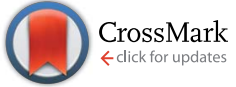

Cite this: RSC Adv., 2015, 5, 83969

Received 8th June 2015

Accepted 28th September 2015

DOI: $10.1039 / \mathrm{c} 5 \mathrm{ra10877b}$

www.rsc.org/advances

\section{Carrier relaxation dynamics in defect states of epitaxial GaN/AIN/Si using ultrafast transient absorption spectroscopy}

\author{
Palak Dugar, ${ }^{a}$ Mahesh Kumar, ${ }^{a}$ Shibin Krishna T. C., ${ }^{\text {ab }}$ Neha Aggarwal ${ }^{\text {ab }}$ \\ and Govind Gupta*ab
}

\begin{abstract}
The relaxation dynamics of the carriers through the defect levels in an epitaxial GaN film grown with an AIN buffer layer on Si has been performed on the femto-picosecond timescale, using ultrafast transient absorption spectroscopy (UFTS). The sample was pumped above and below the band gap and probed with a white light continuum (480-800 nm). A combination of bi and triple exponential decay functions at different probe wavelengths were used to fit the kinetic profile of the carriers in the defect continuum. Based on the UFTS measurements, a model is proposed which explains the dynamics in the shallow traps and deep level defects. Furthermore, to determine the role of the lattice in the relaxation dynamics, the experiment was conducted at a low lattice temperature of $4.2 \mathrm{~K}$. The relaxation constants from the UFTS measurements confirm not only the presence of shallow and deep level defects but also the involvement of phonons in one of the relaxation processes.
\end{abstract}

In the last two decades gallium nitride has inspired a plethora of research activities because of its potential application in optoelectronic and photovoltaic devices such as solar cells, ${ }^{1}$ solar-blind photodetectors, ${ }^{2,3}$ LEDs $^{4-6}$ and laser diodes ${ }^{7,8}$ due to its capability to tailor the band gap through the visible spectrum by alloying with $\mathrm{Al}$ and In. The bulky and toxic nature of the currently available UV lamps has also sparked interest in realizing portable and robust GaN based UV-LEDs. ${ }^{5}$ Such devices employ the UV region for purposes such as water sterilisation, ${ }^{9}$ air purification, surface disinfection, ${ }^{\mathbf{1 0}}$ non-line-of-sight conversion and space to space communication, ${ }^{\mathbf{1 1 , 1 2}}$ counterfeit detection and detectors for particle radiation, biological, ${ }^{\mathbf{1 3 , 1 4}}$ chemical agents ${ }^{\mathbf{1 5}}$ and etc. Furthermore, in many of the above mentioned devices and applications it is imperative to detect UV light without detecting the infrared or visible light, in order to minimize the chances of erroneous detection or high background. ${ }^{\mathbf{1 6 , 1 7}}$ It is well known that wide band gap semiconductors like GaN and $\mathrm{ZnO}$ have inherent defects, ${ }^{\mathbf{1 8}}$ which can significantly alter the optical properties and performance of the device. ${ }^{19-21}$ Park et al. ${ }^{17}$ report the application of a low current blocking layer of AlGaN to minimise erroneous detection. These defect components in the emission spectrum are undesirable and limit the performance of laser devices ${ }^{22}$ and in LEDs, these defects affect the carrier injection. ${ }^{23,24}$ Hence, a detailed analysis

${ }^{a}$ Physics of Energy Harvesting Division, CSIR-National Physical Laboratory (CSIR-NPL), Dr. K.S. Krishnan Marg, New Delhi-110012, India.E-mail: govind@ nplindia.org

${ }^{b}$ Academy of Scientific and Innovative Research (AcSIR), CSIR-NPL Campus, Dr. K.S. Krishnan Marg, New Delhi-110012, India of the non-equilibrium carrier dynamics of GaN films in the visible region is critical not only to understand the carrier transport and relaxation process but also for optimizing the grown structure for fabrication of energy efficient optoelectronic and photovoltaic devices. The availability of ultrafast laser systems which can produce pulses in the range of femtoseconds has enabled the investigation of carrier dynamics and transitions taking place in the femto-picosecond time scale. In the current study of carrier relaxation through UFTS, which is a non-contact method to study the defect dynamics, we have provided complementary information to steady-state experiments in the band-gap region which may not be easily accessible using techniques such as photoluminescence and deep level transient spectroscopy. A lot of work highlighting the femtosecond non-equilibrium dynamics in the near band gap or excitonic region ${ }^{25-30}$ has been carried out. However, in this paper an attempt has been made to analyse carrier dynamics in defect states in the femto-picosecond timescale in an unintentionally doped (UID) $n$-GaN, an inorganic semiconductor in the visible region of the spectrum $(\sim 480-800 \mathrm{~nm})$ based on a spectro-temporal model taking the probe wavelength $(\lambda)$, time elapse after excitation $(t)$ and difference absorption $(\Delta A(\lambda, t))$ as the model parameters. For analysis of the data, the temporal variable ' $t$ ' and the spectral variable ' $\lambda$ ' have been plotted to show the spectral variation of the signal for a given delay time, i.e. an evolution associated difference spectra (EADS) and a plot of the progression of the difference absorption signal $(\Delta A(\lambda, t))$ after $t$ at a given probe wavelength, i.e. a kinetic trace has been obtained. The utilization of a white light continuum probe 
beam and the EADS for a continuous range of probe wavelength offers an in-depth analysis of the defect region provides an insight into the thermalization process of the hot carriers in addition to the spectral and temporal properties of the different processes that govern the relaxation in the GaN film. The UFTS measurements have served as a tool to understand the carrier transport mechanism which enhances functionality by providing information about the sample emission origin.

In this report, the GaN film was grown by plasma assistedmolecular beam epitaxy (Riber Compact 21) equipped with a radio frequency plasma source (Addon) for supplying active nitrogen species and an effusion cell to evaporate $\mathrm{Ga}$ on the substrate. The $\mathrm{Si}(111)$ which is used as a substrate for the growth of GaN film was chemically pre-cleaned by employing the standard RCA cleaning process followed by outgassing in the buffer chamber at $600{ }^{\circ} \mathrm{C}$. The growth chamber pressure of $3.0 \times 10^{-5}$ Torr and RF plasma power of $500 \mathrm{~W}$ was kept constant throughout the growth process. Initially, an AlN buffer layer was grown on an atomically clean $\mathrm{Si}(111) 7 \times 7$ reconstructed surface at $850{ }^{\circ} \mathrm{C}$, followed by the epitaxial GaN film which was grown at $740{ }^{\circ} \mathrm{C}$ with a Ga beam equivalent flux of 8.5 $\times 10^{-7}$ Torr. The epitaxial nature of the grown GaN film was analysed in situ by Reflection High Energy Electron Diffraction (RHEED) using STAIB electron gun operating at $12 \mathrm{keV}$. The structural quality of GaN film was examined by High Resolution X-ray Diffraction (HRXRD, Panalytical X'Pert PRO MRD System). Cross sectional Field Emission Secondary Electron Microscopy (FESEM, ZEISS AURIGA) was employed to measure the thickness of the grown GaN film. The photoluminescence (PL) measurement of the grown sample was performed with a continuous wave excitation of $325 \mathrm{~nm}$ using a He-Cd laser (Melles Griot). The carrier concentration of the grown GaN film was determined by performing Hall measurements and was found to be $1.48 \times 10^{17} \mathrm{~cm}^{-3}$. The UFTS is carried out through the pump-probe method in which the sample is excited at a given wavelength by using the Ti:Sapphire laser and a relatively weaker probe pulse, at a broad range of delay times is used to investigate the relaxation dynamics of the excited carriers. The Ultrafast Transient Absorption Spectrometer (Helios, Ultrafast systems) is integrated to an amplified Ti:Sapphire Laser System (Coherent Legend). The laser system produces a pulse with 800 $\mathrm{nm}, 35$ fs pulse width with $1 \mathrm{kHz}$ repetition rate and $4 \mathrm{~mJ}$ average energy output. Fig. 1 shows a simplified block diagram of the UFTS set-up. The pulse is fed into a cavity containing a stretcher/compressor and regenerative amplifier, pumped by a (VERDI). The output of the regenerative amplifier is again an $800 \mathrm{~nm}, \sim 45 \mathrm{fs}$ pulse with a repetition rate of $1 \mathrm{kHz}$. Approximately $50 \%$ is fed into an Optical Parametric Amplifier-OPA (TOPAS from Light Conversion) for generating the appropriate pump wavelength, while the remaining $10 \%$ is used to generate the white light continuum through a Sapphire disk for the probe pulse. The OPA generates spectrally tunable (240-2600 $\mathrm{nm}$ ) femtosecond pulses with a pulse width of 70 fs to optically pump the sample. In order to obtain the difference spectra $(\Delta A)$ signal, the pump beam frequency was halved to $500 \mathrm{~Hz}$ with a chopper, the sample was then pumped at a fluence of $20 \mu \mathrm{J}$ $\mathrm{cm}^{-2}$. Highly stable output pump beams of 320 and $420 \mathrm{~nm}$ from TOPAS were used to excite the carriers above and below the band-edge, in other words a UV-vis pump and a visible light probe were used to conduct the experiment. For the above mentioned excitations, EADS was taken for a delay time ranging from 1 to 100 ps. To check the dependence of the carrier relaxation time on the lattice temperature, the experiment was performed at a controlled temperature of $4.2 \mathrm{~K}$ by placing the sample into a continuous helium flow cryostat (Optistat CF2). The fitting of the experimental data obtained from UFTS was carried out using Surface Xplorer.

The structural quality of the grown GaN film was analysed using HR-XRD measurement. Fig. 2 shows the $2 \theta$ scan of GaN film grown on $\mathrm{Si}$ (111) substrate. The $2 \theta$ peak positions at $34.75^{\circ}$ and $36.2^{\circ}$ are attributed to diffraction along (0002) plane from GaN and AlN grown film respectively, while the peak at $73.05^{\circ}$ corresponds to the (0004) plane of diffraction of GaN. In addition, two sharp peaks at $2 \theta$ position of $28.85^{\circ}$ and $59.25^{\circ}$ are attributed to (111) and (222) plane of diffraction from silicon substrate. The presence of first and second order X-ray diffractions of GaN in the $2 \theta$ scan confirms the formation of crystalline GaN film grown on silicon substrate. The RHEED pattern observed for GaN along (11-20) and (1-100) zone axes are shown in the inset of Fig. 2 marked as (a) and (b). The typical sharp and streaky 1x1 reconstructed RHEED pattern of GaN film has been observed. The Kikuchi diffraction pattern observed in the (11-

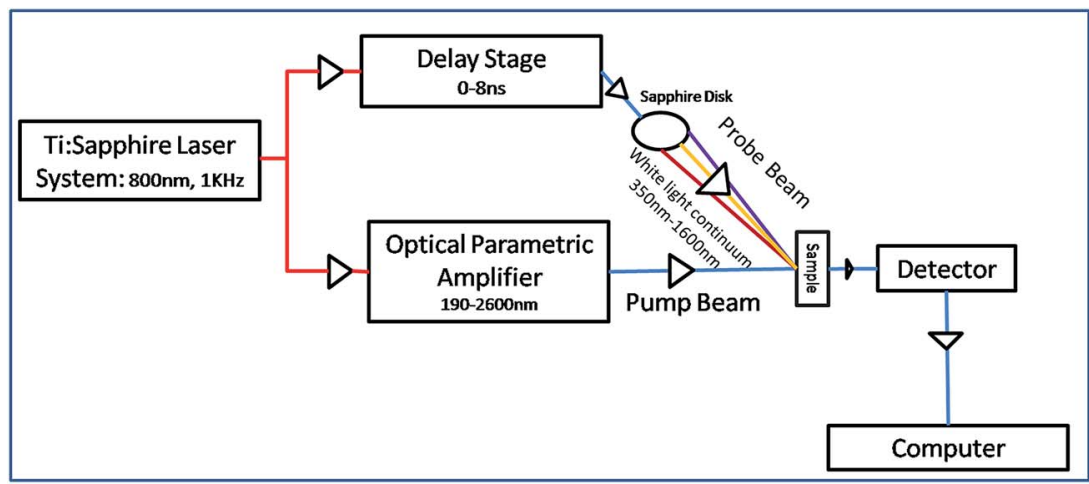

Fig. 1 A simplified block diagram of the ultrafast transient absorption system. 


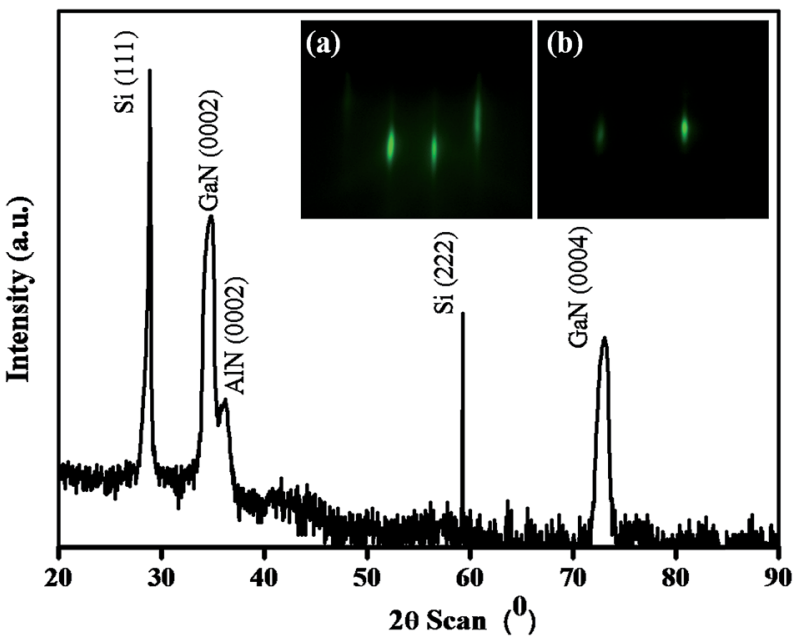

Fig. 2 HR-XRD $2 \theta$ scan of GaN film grown on Si (111) substrate. The RHEED pattern for GaN in the (11-20) and (1-100) zone axes are shown in inset (a) and (b) respectively.

20) direction manifests the two-dimensional (2D) growth of GaN epitaxial film. To establish the optical property of the sample, a PL study was conducted. The PL spectrum for the GaN/Si film is shown in Fig. 3. From the band edge transition peak, the bandgap of the GaN film was determined to be $3.41 \mathrm{eV}$ at RT. The peak at $3.2 \mathrm{eV}$ has been attributed to the transitions from the shallow donor levels due to the unintentional doping of the GaN film. ${ }^{18}$ The presence of a broad hump centred at $\sim 3 \mathrm{eV}$ points towards blue luminescence related defect states. This broad hump is related to transitions from shallow donor levels to deep acceptor levels. ${ }^{31}$ Another broad peak centred at $2.37 \mathrm{eV}$ has been identified as the green luminescence. Since, the sample has been grown with a high Ga flux, therefore the deep acceptor states can be created in the sample due to the excess $\mathrm{Ga}$ and this luminescence is related to internal transitions in defect states. ${ }^{18}$ Carrier relaxation dynamics from these defects states are studied and explored in detail via UFTS in next section. Inset of Fig. 3 shows the cross sectional FESEM image of GaN/AlN/Si

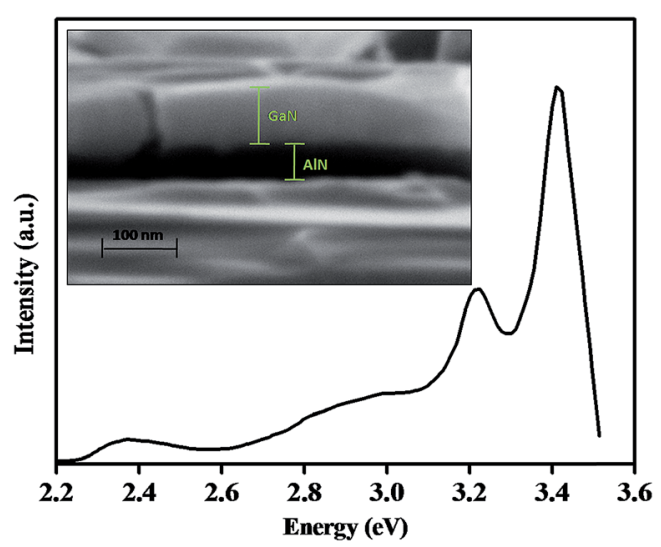

Fig. 3 The RT continuous wave photoluminescence spectrum for the sample. The inset shows the cross-sectional FE-SEM of the sample with the thickness of the AIN layer as $45 \mathrm{~nm}$ and $85 \mathrm{~nm}$ of GaN layer. heterostructure. A legible interface has been observed and the thickness of AlN and GaN films were found to be $45 \mathrm{~nm}$ and 85 $\mathrm{nm}$ respectively with an error of $\pm 10 \mathrm{~nm}$.

In a typical plot of $\Delta A(\lambda, t)$ (optical density) with $\lambda(\mathrm{nm})$ a broad bleaching and a single broad band is recorded. Fig. 4 shows the EADS as a function of probe wavelength, $\lambda$ in the range of $480-800 \mathrm{~nm}$ corresponding to the delay times, $t$ (ps) from $0-100 \mathrm{ps}$ for the sample excited at $320 \mathrm{~nm}$. The figure shows the bleaching states from $480-600 \mathrm{~nm}$ and the excited state absorption from $600-800 \mathrm{~nm}$. The inset (a) of Fig. 4 shows the time profile for the carrier relaxation corresponding to the various wavelengths from 521-771 $\mathrm{nm}$. For perfect fitting, a triple exponential decay model has been used for $\lambda$ at which a negative $\Delta A$ signal is present. The following equation was used for fitting:

$$
S(t)=\mathrm{e}^{-\left(\frac{t-t_{0}}{\operatorname{IRF}}\right)^{2}}\left\{A_{1} \mathrm{e}^{-\left(\frac{t-t_{0}}{\tau_{1}}\right)}+A_{2} \mathrm{e}^{-\left(\frac{t-t_{0}}{\tau_{2}}\right)}+A_{3} \mathrm{e}^{-\left(\frac{t-t_{0}}{\tau_{3}}\right)}\right\}
$$

where, IRF is an instrument response function, $t_{0}$ is time zero, $A_{i}$ and $\tau_{i}$ are amplitudes and decay times respectively. The decay parameters from the triple exponential fit for the bleaching states and the bi-exponential fits for the excited state absorption are shown in Table 1.

While exciting the sample at $320 \mathrm{~nm}$ creates electron-hole pairs above the GaN band edge, the observation of a negative signal at probe wavelengths of 480 to $600 \mathrm{~nm}$ leads to draw an inference that defect levels are present in this region. ${ }^{32,33}$ In view of this, the three time constants have been assigned to three probable mechanisms. In the first mechanism the carriers (37.2-11\%) decay to a mid-gap defect level ${ }^{34}$ with $\tau_{1} \sim 8.06$ to10 ps, in the second mechanism the carriers $(58.2-80.5 \%)$ decay to deep acceptor states near the valence band edge with $\tau_{2} \sim 35.4$ to $28.8 \mathrm{ps}$ and in the third mechanism the carriers (4.67-8.44\%) relax to the valence band edge ${ }^{32}$ with $\tau_{3}$ ranging from 608 ps to 1000 ps as the decay constant. A proposed model highlighting these transitions is shown as inset (b) in Fig. 4. A comparison of the minimum value of $\Delta A$ with $\lambda$ works as an approximation of the density of states at the specific probe wavelengths. ${ }^{32}$ As the minimum $\Delta A$ decreases with $\lambda$, it is inferred that the density of defect states decreases from 480 to $600 \mathrm{~nm}$. Since the detector employed in the experiment is set to measure from 470 to 800 $\mathrm{nm}$ hence a distinct ground state bleaching (GSB) peak is not observed but rather a continuum of a bleaching band can be seen in Fig. 3 for a delay time of $\sim 0.4$ ps to 25 ps. The broadness in the GSB from $480-600 \mathrm{~nm}$ shows that the part of stimulated emission is also present in the GSB band. It can be seen from the onset value of $\Delta A$ for the bleaching band that with an increase in delay time the bleaching decreases with a maximum value at a delay time of $0.5 \mathrm{ps}$, in other words with an increasing delay time, the defect continuum saturates as stimulated emission from the shallow trap levels to the ground state of the sample decreases. The observation of a broad absorption band from $\sim 600$ to $800 \mathrm{~nm}$ can be from two photon absorption (TPA) due to the presence of defect levels in the mid-gap region..$^{32}$ The 


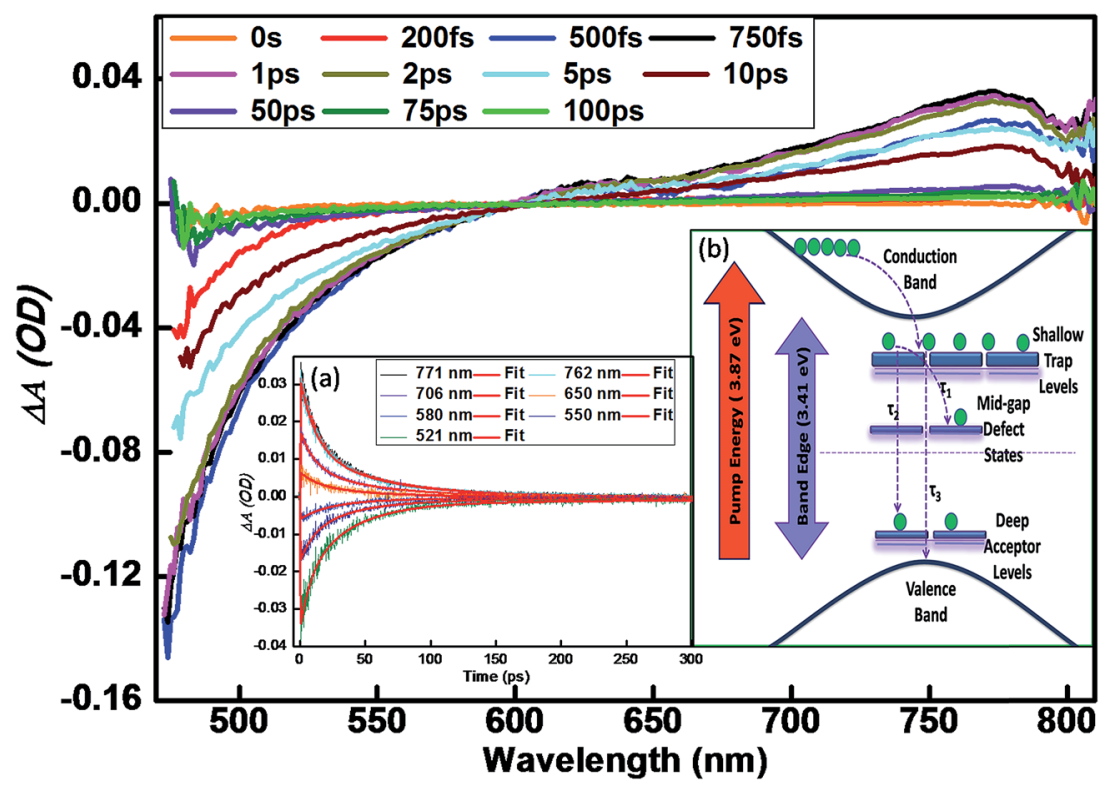

Fig. 4 EADS of the sample excited at $320 \mathrm{~nm}$ for a time delay of 0-100 ps and inset (a) shows the respective kinetic traces at different probe wavelengths for a delay time of 0.750 ps, RT with their fitted decay profile (b) illustrates the proposed model for the corresponding relaxation mechanism.

signal corresponds to the presence of mid-gap defects and indicates that these defect states are populated with a carrier localization time of less than $0.2 \mathrm{ps}$ for all the above $\lambda$. A sequential analysis of Fig. 4 reveals that saturation in the positive signal is achieved at a delay time of $\sim 0.75 \mathrm{ps}$, on either side of which the absorption decreases. For delay times in the range of $0.5-25 \mathrm{ps}$, the absorption band maximum is observed at $\sim 771 \mathrm{~nm}$ which corresponds to an energy of $1.61 \mathrm{eV}$. For $\lambda$ ranging from $600-800 \mathrm{~nm}$, a perfect fit was obtained for the band with two relaxation time constants, $\tau_{1}$ and $\tau_{2}$ as shown in Table 1. A systematic analysis is required to establish the correlation between specific defects such as screw and edge threading dislocations, point defects, grain boundaries etc. to the particular defect levels in the forbidden gap.

For a better understanding of the above mentioned defect levels and their relaxation mechanisms, the carriers were excited at $420 \mathrm{~nm}$ to populate the shallow levels below the band gap (480-600 nm). The time resolved absorption of the sample at specific wavelengths is shown in Fig. 5 . The inset (a) of Fig. 5 shows the EADS spectrum at various time delays in which only a positive signal for $\lambda$ from 480 to $800 \mathrm{~nm}$ is observed with a broad hump centred at $480-650 \mathrm{~nm}$ and a broad peak at $\sim 771 \mathrm{~nm}$. A triple exponential decay yields a perfect fit for all the probe wavelengths. A schematic model for the relaxation process is shown in Fig. 5, inset (b). It is proposed that the initially hot carriers (84.3-57.8\%) scatter into the available bands with a relaxation time, $\tau_{1}$ ranging from $0.154-1.88$ ps. It is inferred from the gradual increase in $\tau_{1}$ with the probe wavelength that as the energy of the generated carriers decreases the number of available states for scattering decreases. These carriers (14.1$34 \%)$ then either relax to mid gap defect levels with $\tau_{2}$ as the relaxation time which is in the range of 3.45 ps to 16.7 ps or they relax to the ground state in the valence band region with a long decay time of $\tau_{3}(1.66-8.19 \%)$ from $554 \mathrm{ps}$ to $1890 \mathrm{ps}$. As shown in Table 1, the amplitude corresponding to the first two decay constants, $\tau_{1}$ and $\tau_{2}$ contribute to the positive signal and the amplitude of the third, $\tau_{3}$ is responsible for the negative dip observed in the absorption signal of Fig. 5 .

To ascertain the level of interaction of the carriers with the lattice, the UFTS measurements were carried out at the same

Table 1 Fitting parameters for excitation at $320 \mathrm{~nm}$ (RT and $4.2 \mathrm{~K}$ ) and $420 \mathrm{~nm}$

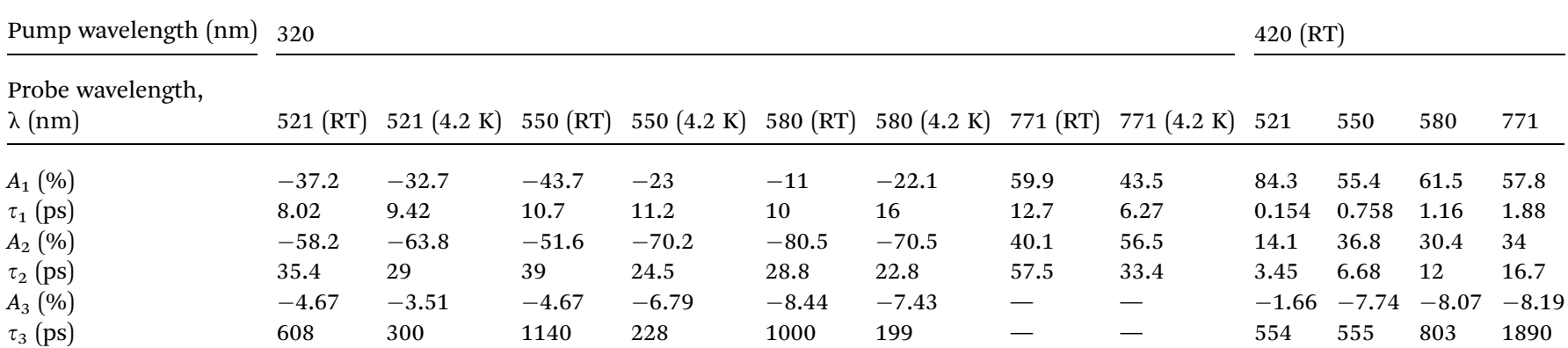




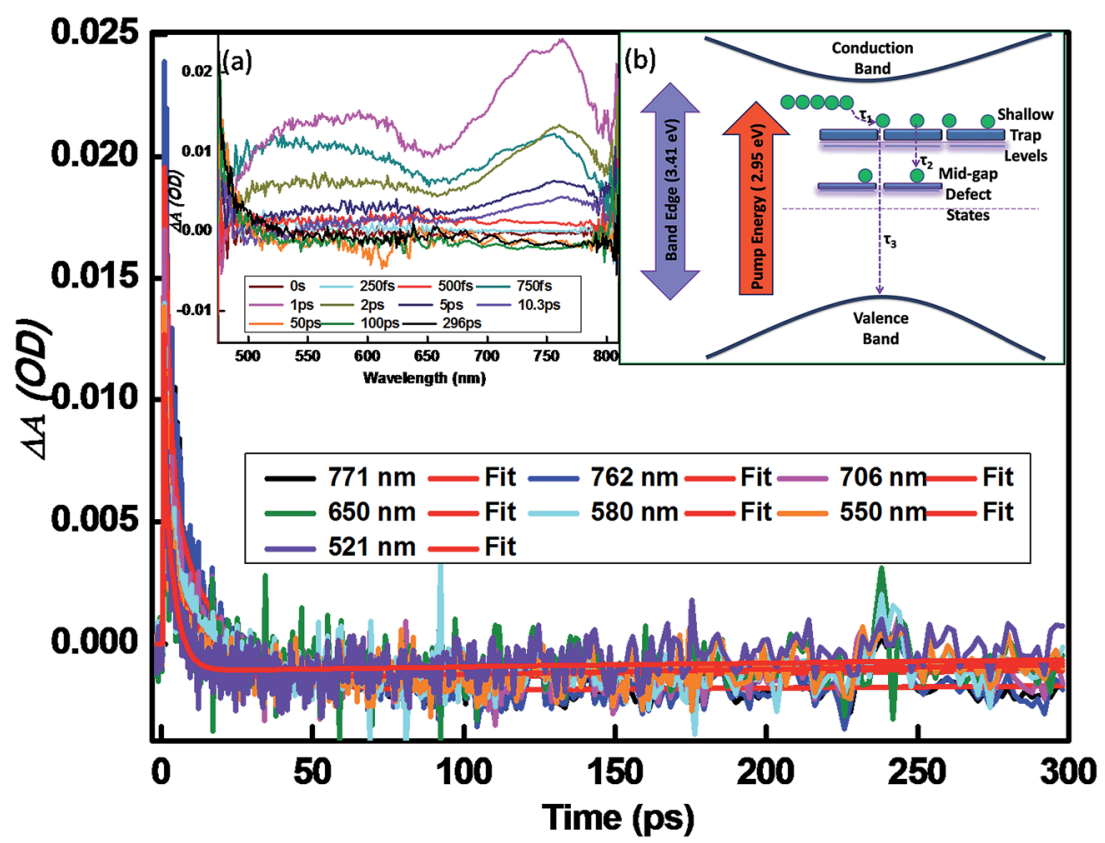

Fig. 5 The kinetic traces for below band gap excitation of $420 \mathrm{~nm}$ at different probe wavelengths for a delay time of 1 ps, RT with their fitted decay profile. Inset (a) shows the EADS of the sample excited at $420 \mathrm{~nm}$ for a time delay of $0-100 \mathrm{ps}$. (b) Illustration of the relaxation mechanism for pumping at $420 \mathrm{~nm}$.

fluence and pump excitation of $320 \mathrm{~nm}$ at $4.2 \mathrm{~K}$. Fig. 6 shows the EADS of the sample for maximum signal at RT and $4.2 \mathrm{~K}$. The band filling effect can be seen till $636 \mathrm{~nm}$ at $4.2 \mathrm{~K}$ as compared to $600 \mathrm{~nm}$ at RT. In addition to this, two distinctive features can be seen at $4.2 \mathrm{~K}$. The first is the presence of a peak centred at $760.6 \mathrm{~nm}(1.63 \mathrm{eV})$ and the second is the decrease in magnitude of the positive absorption band till $786 \mathrm{~nm}$ followed by an increased $\Delta A$ signal (near infrared region) relative to RT. The parameters obtained at $4.2 \mathrm{~K}$ fits well with the model outlined in Fig. 4, inset (b). On comparing the decay

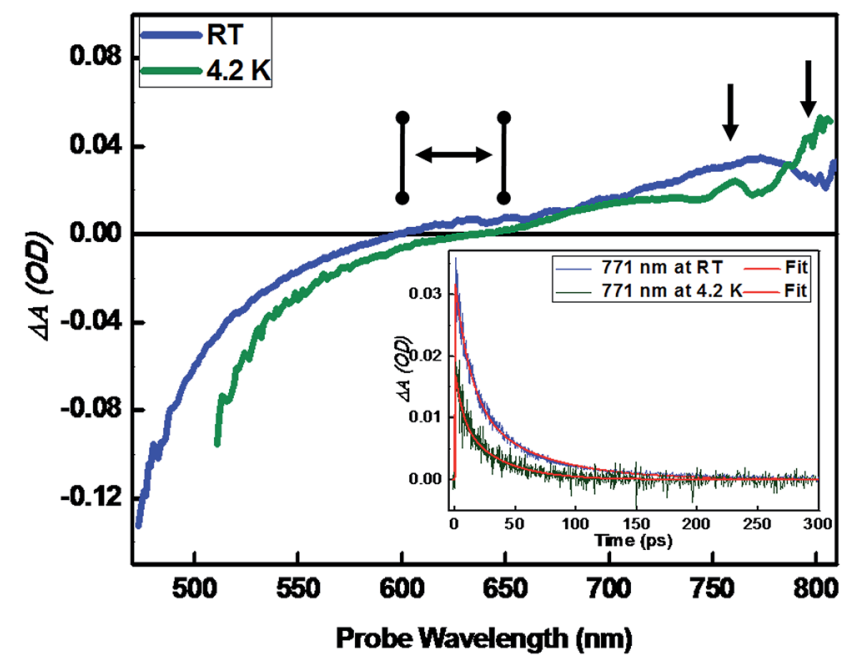

Fig. 6 The EADS at which maximum signal is obtained for RT and $4.2 \mathrm{~K}$ and the inset shows the kinetic trace at a probe wavelength of $771 \mathrm{~nm}$. The arrows show the three significant differences that can be seen at $4.2 \mathrm{~K}$ from RT. values listed in Table 1 , for $\lambda(480-636 \mathrm{~nm})$ a considerable decrease in $\tau_{2}$ and $\tau_{3}$ and an increase in the value of $\tau_{1}$ is observed. It can be inferred that the relaxation mechanism associated with $\tau_{1}$ is phonon assisted ${ }^{35}$ whereas, the process related to $\tau_{2}$ and $\tau_{3}$ may take place via carrier-carrier scattering. For $\lambda$ from 636 to $800 \mathrm{~nm}, \tau_{1}$ and $\tau_{2}$ both decrease and in addition to this, the relative number of carriers which relax via the two mechanisms is interchanged. For pumping above band gap, it can be concluded that the mechanism $\tau_{1}$ is a nonradiative process for probe wavelengths where GSB is observed. However, for a definite categorization of the type of process involved with $\tau_{2}$ and $\tau_{3}$ fluence dependent measurements need to be performed.

In summary, UFTS using femtosecond laser pulse was performed on an UID GaN/AIN/Si, this enabled a detailed analysis of the non-equilibrium carrier dynamics in the defect states namely, the shallow trap, mid-gap and deep acceptor (near valence band-edge) levels. The dynamics were studied in detail with the lattice temperature at RT and $4.2 \mathrm{~K}$. For pump excitation of $320 \mathrm{~nm}$, the analysis reveals the presence of shallow defect levels lying in the region of 480 to $600 \mathrm{~nm}$. It also shows excited state absorption from 600 to $800 \mathrm{~nm}$. The bi and triple exponential fits for the relaxation dynamics yields two and three time constants and their respective amplitude contribution to the difference absorption. Based on these parameters a model highlighting the carrier dynamics has been proposed. To support the proposed model, another pump excitation of 420 $\mathrm{nm}$ was used in which the carriers were excited just above these shallow defect levels, from which carrier relaxation was studied. The decay constants obtained from the triple exponential fit for the kinetic trace corroborates the model of shallow and deep level defects. 


\section{Acknowledgements}

This work is financially supported by Council of Scientific and Industrial Research, India under the XII ${ }^{\text {th }}$-FYP-PSC-0109. The authors are thankful to Prof. Anirban Bhattacharyya, University of Calcutta for the photoluminescence measurement. One of the co-author (SKTC) would like to thank DST (Government of India) and SIMCO Global Tech. \& System Ltd. for financial support provided under the Prime Minister Doctoral Research Fellowship.

\section{References}

1 E. Matioli, C. Neufeld, M. Iza, S. C. Cruz, A. A. Al-Heji, X. Chen, R. M. Farrell, S. Keller, S. DenBaars, U. Mishra, S. Nakamura, J. Speck and C. Weisbuch, High internal and external quantum efficiency InGaN/GaN solar cells, Appl. Phys. Lett., 2011, 98(2), 021102.

2 G. Parish, S. Keller, P. Kozodoy, J. P. Ibbetson, H. Marchand, P. T. Fini, S. B. Fleischer, S. P. DenBaars, U. K. Mishra and E. J. Tarsa, High-performance (Al, Ga) N-based solar-blind ultraviolet $\mathrm{p}-\mathrm{i}-\mathrm{n}$ detectors on laterally epitaxially overgrown GaN, Appl. Phys. Lett., 1999, 75(2), 247-249.

3 M. L. Lee, Y. H. Yeh and S. J. Tu, Solar-Blind pin Photodetectors Formed on-Patterned n-GaN Templates, IEEE J. Quantum Electron., 2012, 48(10), 1305-1309.

4 S. Nakamura, T. Mukai and N. Iwasa, Light-emitting gallium nitride-based compound semiconductor device, U.S. Pat. 5,747,832, issued May 5, 1998.

5 W. Guo, M. Zhang, A. Banerjee and P. Bhattacharya, Catalystfree InGaN/GaN nanowire light emitting diodes grown on (001) silicon by molecular beam epitaxy, Nano Lett., 2010, 10(9), 3355-3359.

6 Y. Tchoe, J. Jo, M. Kim, J. Heo, G. Yoo, C. Sone and G. C. Yi, Variable-Color Light-Emitting Diodes using GaN Microdonut arrays, Adv. Mater., 2014, 26(19), 3019-3023.

7 S. Nakamura, S. Pearton and G. Fasol, The blue laser diode: the complete story, Springer Science \& Business Media, 2000.

8 J. W. Raring, E. M. Hall, M. C. Schmidt, C. Poblenz, B. Li, N. Pfister, D. F. Feezell, R. Craig, J. S. Speck, S. P. DenBaars and S. Nakamura, High-power high-efficiency continuouswave InGaN laser diodes in the violet, blue, and green wavelength regimes, Proc. SPIE 7602, Gallium Nitride Materials and Devices V, 2010, vol. 760218, DOI: 10.1117/12.840783.

9 M. A. Würtele, T. Kolbe, M. Lipsz, A. Külberg, M. Weyers, M. Kneissl and M. Jekel, Application of GaN based ultraviolet-C light emitting diodes UV LEDs for water disinfection, Water Res., 2011, 45(3), 1481-1489.

10 M. S. Shur and R. Gaska, Deep-ultraviolet light-emitting diodes, IEEE Trans. Electron Devices, 2010, 57(1), 12-25.

11 S. C. Shen, Y. Zhang, D. Yoo, J. B. Limb and J. H. Ryou, Performance of deep ultraviolet GaN avalanche photodiodes grown by MOCVD, IEEE Photonics Technol. Lett., 2007, 19(21), 1744-1746.

12 A. Katz, B. Eggleston and J. Macdonald, GaN SSPA for UHF space applications, in Microwave Symposium Digest (IMS), 2013 IEEE MTT-S International, IEEE, 2013, pp. 1-4.
13 J. P. Grant, Ph.D thesis, University of Glasgow, 2007.

14 I. H. Lee, A. Y. Polyakov, N. B. Smirnov, A. V. Govorkov, E. A. Kozhukhova, V. M. Zaletin, I. M. Gazizov, N. G. Kolin and S. J. Pearton, Electrical properties and radiation detector performance of free-standing bulk $n$-GaN, J. Vac. Sci. Technol., B: Nanotechnol. Microelectron.: Mater., Process., Meas., Phenom., 2012, 30(2), 021205.

15 R. Kleindienst, M. Krüger, A. Grewe, S. Sinzinger, K. Holc, J. Schätzle, A. Steffen, V. Cimalla, U. T. Schwarz, P. Becker, P. Hille, J. Schörmann, J. Teubert and M. Eickhoff, Integrated Opto-Chemical Sensor System based on Group III-nitride Nanowires, GMM-Fachbericht-Mikro-NanoIntegration, 2014.

16 M. Razeghi and A. Rogalski, Semiconductor ultraviolet detectors, J. Appl. Phys., 1996, 79(10), 7433-7473.

17 K. Y. Park, H. M. Kim, K. H. Lee, S. H. Lee and H. K. Kim, Semiconductor photo-detecting device, U.S. Pat. Application 14/496,998, filed September 25, 2014.

18 M. A. Reshchikov and H. Morkoc, Luminescence properties of defects in GaN, J. Appl. Phys., 2005, 97(6), 061301061395.

19 S. J. Pearton, J. C. Zolper, R. J. Shul and F. Ren, GaN: Processing, defects, and devices, J. Appl. Phys., 1999, 86(1), 1-78.

20 M. Mori, S. Kondo, S. Yamamoto, T. Nakao, T. Fujii, M. Iwaya, T. Takeuchi, S. Kamiyama, I. Akasaki and H. Amano, Correlation between Device Performance and Defects in GaInN-Based Solar Cells, Appl. Phys. Express, 2012, 5(8), 082301.

21 K. Zhang, S. J. Kim, Y. Zhang, T. Heeg, D. G. Schlom, W. Shen and X. Pan, Epitaxial growth of ZnO on (1 111 1) Si free of an amorphous interlayer, J. Phys. D: Appl. Phys., 2014, 47(10), 105302.

22 C. S. Kim, Y. D. Jang, D. M. Shin, J. H. Kim, D. Lee, Y. H. Choi, M. S. Noh and K. J. Yee, Estimation of relative defect densities in InGaN laser diodes by induced absorption of photoexcited carriers, Opt. Express, 2010, 18(26), 27136-27141.

23 Y. Li, F. Yun, X. Su, S. Liu, W. Ding and X. Hou, Carrier injection modulated by V-defects in InGaN/GaN multiplequantum-well blue LEDs, Jpn. J. Appl. Phys., 2014, 53(11), 112103.

24 J. D. Poplawsky, A. Nishikawa, Y. Fujiwara and V. Dierolf, Defect roles in the excitation of $\mathrm{Eu}$ ions in Eu:GaN, Opt. Express, 2013, 21, 30633-30641.

25 Y. C. Chang, C. K. Choi and J. J. Song, Theoretical modeling of femtosecond pump-probe spectroscopy in GaN systems, Proc. SPIE 4280, Ultrafast Phenomena in Semiconductors $V$, 2001, vol. 58.

$26 \mathrm{H}$. Ye, G. W. Wicks and P. M. Fauchet, Hot electron relaxation time in GaN, Appl. Phys. Lett., 1999, 74(5), 711713.

27 C. Y. Yang, C. T. Chia, H. Y. Chen, S. Gwo and K. H. Lin, Ultrafast carrier dynamics in GaN nanorods, Appl. Phys. Lett., 2014, 105(21), 212105.

28 Ü. Özgür and H. O. Everitt, Ultrafast carrier relaxation in GaN, $\operatorname{In}_{0.05} \mathrm{Ga}_{0.95} \mathrm{~N}$, and an $\operatorname{In}_{0.07} \mathrm{Ga}_{0.93} \mathrm{~N} / \mathrm{In}_{0.12} \mathrm{Ga}_{0.88} \mathrm{~N}$ 
multiple quantum well, Phys. Rev. B: Condens. Matter Mater. Phys., 2003, 67(15), 155308-155316.

29 C. K Choi, Y. H. Kwon, J. S. Krasinski, G. H. Park, G. Setlur, J. J. Song and Y. C. Chang, Ultrafast carrier dynamics in a highly excited GaN epilayer, Phys. Rev. B: Condens. Matter Mater. Phys., 2001, 63(11), 115315-115320.

30 A. Kar, Q. Li, P. C. Upadhya, M. A. Seo, J. Wright, T. S. Luk, G. T. Wang and R. P. Prasankumar, The influence of radial heterostructuring on carrier dynamics in gallium nitride nanowires, Appl. Phys. Lett., 2012, $101(14), 143104$.

31 M. A. Reshchikov, F. Shahedipour, R. Y. Korotkov, B. W. Wessels and M. P. Ulmer, Photoluminescence band near $2.9 \mathrm{eV}$ in undoped GaN epitaxial layers, J. Appl. Phys., 2000, 87(7), 3351-3354.
32 D. Tsokkou, A. Othonos and M. Zervos, Defect states of chemical vapor deposition grown GaN nanowires: Effects and mechanisms in the relaxation of carriers, J. Appl. Phys., 2009, 106, 054311-054316.

33 P. C. Upadhya, J. A. Martinez, Q. Li, G. T. Wang, B. S. Swartzentruber, A. J. Taylor and R. P. Prasankumar, Space-and-time-resolved spectroscopy of single GaN nanowires, Appl. Phys. Lett., 2015, 106(26), 263103.

34 H. Haag, B. Hönerlage, O. Briot and R. L. Aulombard, Influence of defect states on the nonlinear optical properties of GaN, Phys. Rev. B: Condens. Matter Mater. Phys., 1999, 60(16), 11624-11630.

35 A. Othonos, Probing ultrafast carrier and phonon dynamics in semiconductors, J. Appl. Phys., 1998, 83(4), 1789-1830. 